\title{
Auf der Suche nach dem verschwiegenen Reichtum
}

\author{
Searching for Clandestine Wealth
}

\section{Martin Schürz*}

\section{Einleitung}

Zum Reichtum gehört der Vergleich. Ein Hedgefondsmanager, dessen private Boeing keinen Whirlpool hat, mag sich arm fühlen im Vergleich zu Milliardär|inn|en. Allein dies erlaubt noch nicht, den Reichtumsbegriff in den Bereich der Gefühle und persönlichen Empfindungen zu exilieren.

Reichtum ist ein Begriff, der von sozialen Deutungen abhängt. Das akademische Interesse in der Wirtschaftsforschung an den Reichen ist relativ jung. ${ }^{\mathrm{I}}$ Es gibt keine Volksvermögensrechnung. Makrodaten (Volkswirtschaftliche Gesamtrechnung, Gesamtwirtschaftliche Finanzierungsrechnung) geben nur Aufschluss über die Entwicklung der einzelnen Sektoren (Haushalte, Unternehmen, Staat), aber nicht über die Vermögensverteilung. Seit vermehrt Mikrodaten zum privaten Haushaltsvermögen existieren, ist die Heterogenität des privaten Haushaltssektors leicht zu erkennen. ${ }^{2}$

Ein Anstieg der sozialen Ungleichheit und ein steigendes Ungerechtigkeitsempfinden in der Bevölkerung erhöhen auch das öffentliche Interesse an Daten zur Vermögensverteilung (vgl. Ehrenreich 2007). Armuts- und Reichtumsberichte reflektieren dieses öffentliche Interesse am Thema (vgl. Deutsche Bundesregierung 2005). Im I. deutschen Armuts- und Reichtumsbericht 200I war der Versuch unternommen worden, ein umfassendes Bild der sozialen Unterschiede in Deutschland zu zeichnen. Jedoch waren lediglich 30 von über 290 Seiten den Vermögenden gewidmet. Eine Analyse über Herkunft und Auswirkungen des Reichtums findet sich nicht. Für den geplanten dritten Bericht 2008 setzt nun zögerlich eine methodische Diskussion zur Reichtumsforschung ein (vgl. ISG 2007).

Konzeptuelle Fragen des Reichtums müssen ausführlich analysiert werden, da Debatten zu den Reichen stets einem Sozialneidvorwurf, Klassenkampfverdacht oder einer Kritik an vorgeblich fehlender Begriffsschärfe (»Ich bin wohlhabend, aber nicht reich») ausgesetzt sind. Wer gegen Reichtum moralisiert und nicht argumentiert, riskiert, bereits mit dem

I Für extensive Diskussionen vgl. Huster (1997), Stadlinger (Hg.) (200I), Mäder/Streuli (2002) und Atkinson (2006).

2 In einer Reihe von Ländern werden regelmäßig Haushaltsbefragungen zum privaten Vermögen durchgeführt. Für exemplarische Länderstudien vgl. Faik (200I), Klevmarken (2003), Beer et al. (2006), Kennickell (2006), Grabka/Frick (2007) und Davies et al. (2007a und 2007b).

* Oesterreichische Nationalbank, Wien. Für wertvolle Kommentare danke ich Willi Altzinger und Pirmin Fessler.

(C) INTERVENTION 5 (I), 2008, 63-76 
Hinweis auf die - besonders in den USA verbreitete - karitative Tätigkeit mancher Reicher argumentativ widerlegt zu werden. Konzeptuelle Fragen zur Armut sind zwar ebenso wertend wie Reichtumsfragen, aber gesellschaftlich konsensualer. ${ }^{3}$

Die fehlende empirische Datenbasis zu Reichtum zu beklagen, ist common sense in der ökonomischen Literatur. ${ }^{4}$ Seltener wird untersucht, wie Reichtum zu bestimmen ist. Volkert (2005) argumentiert für eine Mehrdimensionalität hinsichtlich der Reichtumsvariablen. Doch auch dies ersetzt nicht die begriffliche Bestimmung von Reichtum. Eine Reichtumskonzeption sollte jedenfalls die Liste der notwendigen Daten prägen und nicht die Datenverfügbarkeit die dann untersuchten Problemstellungen.

In diesem Artikel wird der Versuch unternommen, normative Überlegungen bei methodischen Fragestellungen in Zusammenhang mit Reichtum zu diskutieren. Im ersten Teil werden unterschiedliche Kriterien und Indikatoren von Reichtum erörtert. Es soll gezeigt werden, dass die klassische Frage, wer die Reichen sind, zu kurz greift. Der zweite Teil untersucht Möglichkeiten der wissenschaftlichen Erforschung von Reichtum. Argumentiert wird für eine explizite normative Ausrichtung am politischen Gleichheitsideal der Gesellschaft. Dies junktimiert dann Reichtum und Armut. Die Beantwortung der Fragen, wie die Reichen reich wurden, und was die Reichen mit ihrem Reichtum tun, erlaubt eine normativ gehaltvolle Konzeption von arm machendem Reichtum.

\section{Kriterien von Reichtum}

Gemeinhin gelten Millionär|inn|e|n als reich. Zwischen Einkommensmillionär|inn|en und Vermögensmillionär|inn|en wird medial nur selten unterschieden und die Bezeichnung ist eher von kultureller Bedeutung, da sie von der Kaufkraft der jeweiligen Währung abhängt (vgl. Huster 2007). ${ }^{5}$

Definitionen analog den Armutsdefinitionen sind beim Reichtum schwierig. Gibt es einen absoluten oder relativen Reichtum, einen Erwerbsreichtum? Und was wäre primärer Reichtum bzw. eine Reichtumslücke?

Im Bereich der finanziellen Ressourcen können recht willkürlich definierte Reichtumsschwellenwerte unterschieden werden. Vermögensreichtum wird im deutschen Armutsund Reichtumsbericht u. a. über den Anteil derjenigen Personen bestimmt, deren Vermögenseinkommen ein Vielfaches des durchschnittlichen Äquivalenzeinkommens beträgt (Huster 200I: I4). Diese Definition fokussiert implizit auf gehobenen Konsum und finanzielle Unabhängigkeit. Was unter gehobenem Konsum zu verstehen ist, bleibt aber ungeklärt. Über den Luxuskonsum kann Reichtum in einer Konsumgesellschaft nur unzulänglich gemessen werden. Der Besitz eines Sportwagens ist auch für Personen mit mittleren

3 Die Position, arme Menschen einfach verhungern zu lassen, wird etwa von niemandem vertreten. Auch dies zeigt die Asymmetrie in der Analyse von Armut und Reichtum.

4 Exemplarisch vgl. Davies/Shorrocks (2000), Stein (2004) und Deutsche Bundesregierung (2005).

5 Aber auch die Währungsumstellung zum Euro zeigt die Problematik des gebräuchlichen Millionär|innenbildes deutlich, weil ein|e DM-Millionär|in noch kein|e Euromillionär|in sein muss. 
Einkommen möglich, sofern sie dafür auf andere Güter verzichten. Und wie viele Austern die Reichen schlürfen, bleibt unerheblich.

Setzt man die Reichtumsgrenzen auf 200 Prozent oder 300 Prozent des äquivalenzgewichteten Einkommens, wären in Deutschland 7,I Prozent bzw. I,5 Prozent der Bevölkerung reich. Reich würde hier einkommensstark bzw. höher verdienend bezeichnen. Doch nicht nur die Wahl des Faktors zwei bzw. drei ist arbiträr, auch die durchgängige Verwendung der OECD-Äquivalenzskala würde zu absurden Ergebnissen führen. Ein kleines Kind in einer dreiköpfigen Millionärsfamilie würde mit einem sechsstelligen Euro-Mehrbedarf in die Kalkulation eingehen. ${ }^{6}$ Die Äquivalenzskala zielt nur auf Bedarf aus einer Armutsperspektive. Im oberen Einkommensbereich geht die Aussagekraft vollständig verloren.

Weit höhere Grenzen könnte man dort ziehen, wo zunehmender Reichtum nicht mehr der Steigerung des Lebensstandards, sondern der weiteren Vermögensbildung dient. Dies markiert den Unterschied zwischen wohlhabend und reich. High net worth individuals (HNWIs) sind Personen mit Nettogeldvermögen von mehr als einer Million US-\$. Der Studie » Global Wealth 2007« von Merrill Lynch / Capgemini (2007) zufolge gibt es derzeit weltweit 9,6 Millionen Haushalte über diesem Schwellenwert (ohne Immobilienvermögen).

Um einer willkürlichen Bestimmung der Reichtumsgrenze auszuweichen, werden oft Anteile von Quantilen am gesamten Nettovermögen graphisch dargestellt und ihre Veränderung im Zeitablauf illustriert. ${ }^{7}$ Die Willkürlichkeit in der Auswahl bleibt aber bestehen, da zwischen Perzentilen, Dezilen und Quintilen ausgewählt werden kann. Im Zeitablauf ergeben sich in den einzelnen Intervallen der Vermögensverteilung oft unterschiedliche Entwicklungen.

Eine Vielfalt von Reichtumsdefinitionen ist nicht problematisch, solange keine wirtschaftspolitischen Maßnahmen - wie etwa die Erhebung einer Vermögen- oder Erbschaftssteuer - daraus abgeleitet werden. Implizite normative Wertungen mögen sich in den Präferenzen der Forscher|innen verbergen, die Zahl der Reichen klein bzw. groß zu halten, aber die Daten selbst geben keine Handlungsempfehlungen. Wird die Reichtumsschwelle analog der Armutsdefinition gelegt, dann wird Reichtum zu einem Massenphänomen. ${ }^{8}$ Bezieht man sich nur auf die Super-Reichen, so ist man nahe am Thema der Vermögenskonzentration.

Ab welchem Schwellenwert von Reichtum gesprochen werden kann, ist nicht so wichtig wie die Frage, was Reichtum überhaupt ausmacht. Entsprechend dem Fähigkeitenansatz von Amartya Sen wird Armut als Mangel an Verwirklichungschancen verstanden. Und einer Interpretation zufolge wäre Reichtum eine Vielzahl an Verwirklichungschancen (vgl. Volkert 2005). Eine solche Interpretation bleibt auf die positiven Funktionen von Reichtum

6 Das Nettoäquivalenzeinkommen einer Person ergibt sich aus dem Haushaltsnettoeinkommen dividiert durch die Summe der Personengewichte der Haushaltsmitglieder. Die OECD-Äquivalenzskala misst dem Haushaltsvorstand ein Gewicht von eins zu, weiteren Personen des Haushalts über I4 Jahren ein Gewicht von 0,5 und Kindern unter I4 Jahren 0,3.

7 Für Darstellungen zur Vermögensverteilung im 20. Jahrhundert vgl. Atkinson et al. (1989), Kopczuk/Saez (2004) und Piketty et al. (2006).

8 Die Vermögensverteilung ist nach unten begrenzt (Einkommens-, Kreditbeschränkungen) und nach oben offen. 
konzentriert und greift analytisch meines Erachtens zu kurz, da sie gerade das Übermaß an Verwirklichungschancen reicher Menschen verfehlt. Für den französischen Soziologen Pierre Bourdieu umfasst Reichtum verschiedene Formen von Kapital (ökonomisches Kapital, Bildung und soziale Netze) innerhalb eines bestimmten Feldes (vgl. Schürz 2008a).

\section{Indikatoren der Reichtumsmessung?}

Als Indikatoren des Reichtums können Einkommen, Vermögen, menschliche Entwicklung 9 oder Fähigkeiten herangezogen werden. Die Vermögensungleichheit ist weit größer als die Einkommensungleichheit und Vermögen ist ein Indikator von Macht und von langfristiger ökonomischer Sicherheit. Daher wird Vermögen für eine Reichtumsbetrachtung wichtiger sein als Einkommen. Vermögensreichtum unterscheidet sich von Einkommensreichtum durch die größere Dauerhaftigkeit.

Bei Vermögen kann zwischen Produktivvermögen, Grundvermögen, Geldvermögen (Spareinlagen, Anleihen, Beteiligungswerte), immateriellem Vermögen (Lizenzen, Urheberrechte, Patente), Naturvermögen, Humanvermögen (Qualifikation der menschlichen Arbeitskraft) und Sozialvermögen (Pensionsansprüche) unterschieden werden (vgl. Schürz 2008b). Für Reichtumskonzentration ist besonders Produktivvermögen von Bedeutung. Reichtum erstreckt sich realiter aber - im Gegensatz zum isolierten Geldgewinn beim Glücksspiel - über verschiedene Vermögensformen. ${ }^{\text {To }}$

Vermögen erfüllt generell zahlreiche Funktionen:

(I) Einkommenserzielungsfunktion: Vermögen erbringt Einkommen in Form von Dividenden, Mieten, Pachten, Zinsen oder ausgeschütteten Gewinnen.

(2) Nutzenfunktion: Sachvermögen kann für Produktions- und Konsumzwecke verwendet werden.

(3) Sicherungsfunktion: Vermögen kann in Notfällen verwendet werden.

(4) Transformationsfunktion: Vermögen kann auf verschiedene Zeitperioden verschoben werden (Erben und Schenken).

(5) Machtfunktion: Größere Vermögen verleihen gesellschaftlichen Status und politischökonomische Macht.

Einige dieser Funktionen können für alle Vermögensbesitzer|innen wichtig sein (Einkommenserzielung und Nutzung). Andere sind vorrangig für die Reichen (Vererbung, Macht und Status) und wieder andere insbesondere für einkommensarme Menschen (Notgroschen) entscheidend. Nur die ersten drei Funktionen stehen im Zentrum der ökonomischen Forschung (vgl. Davies/Shorrocks 2000). Die Untersuchung jener Vermögensfunktionen,

9 UNDP-Entwicklungsindikator Human Development Indicator (HDI; arithmetisches Mittel des Logarithmus des Einkommens pro Kopf, der Lebenserwartung und der Bildung).

Io In der Regel wird Immobilienvermögen erst ab einem höheren Geldvermögensbestand erworben und (direktes) Unternehmenseigentum ist besonders in den höchsten Vermögensstufen von Relevanz. 
die für die Reichen von Interesse sind, wird in der ökonomischen Literatur fast vollständig vernachlässigt. ${ }^{\text {II }}$ Doch gibt es interessante Verschränkungen zwischen verschiedenen Kapitalformen. Ermisch et al. (2006) zeigen etwa auf Basis von britischen British Household Panel Survey (BHPS)- und deutschen Socio-economic Panel Study (SOEP)-Daten, dass das Heiratsverhalten entlang der sozialen Positionen läuft, Reiche demnach Reiche heiraten.

Die medialen Schätzungen zum Vermögen der Reichen werden breit rezipiert, doch die Datenqualität kann nicht überprüft werden. Vernachlässigt werden oft bestimmte Formen des Vermögens und geachtet wird zu sehr auf die Aktivseite und zu wenig auf die Verschuldung (vgl. Atkinson 2006). ${ }^{\text {22 }}$

Eine klassische Methode der Sozialforschung basiert aufTiefeninterviews, wo Motivationen zur Vermögensakkumulation und Einstellungen zum Vermögen untersucht werden können. Der Preis für die Verwendung dieser Methode dürfte dann hoch sein, wenn vertrauliche Informationen nur im Austausch gegen Loyalität der Forscher|innen gegeben werden: Mit anderen Worten, wenn die Sozialforscher|innen von den Reichen entweder als ihrer Schicht zugehörig oder wenigstens als ihnen ideologisch gewogen wahrgenommen werden müssen, um an Informationen zu gelangen. Eine Forschergruppe um Ueli Mäder untersucht Reichtum in der Schweiz mittels Tiefeninterviews und diagnostizierte Diversität bei den Reichtumstypen (vgl. Mäder et al. 2002).

In der Oesterreichischen Nationalbank (OeNB) wird aktuell versucht, milieugestützte Wahrnehmungen zu Vermögen mittels Gruppendiskussionen zu eruieren und die Ergebnisse unterschiedlicher Milieus (Langzeitarbeitslose, Student|inn|en, Frauen usw.) miteinander zu kontrastieren. Erste Ergebnisse zeigen eine Vielfalt von Reichtumsbildern. Ein mythisch aufgeladener und ein lebensweltlich verankerter Reichtumsbegriff markieren die Pole. Reich sei, wer nicht mehr wisse, wie er|sie sein|ihr Vermögen ausgeben könne; oder, reich sei, in der bescheidenen Variante, wer eine Wohnung und ein Autos sein|ihr Eigen nenne. Beide Konnotationen haben nur wenig miteinander zu tun. Die Vorstellungen von erreichbarem Reichtum dürften materialistisch in den realen Möglichkeiten von Menschen verankert sein. Arme Menschen entwickeln oft nicht einmal Phantasien über Reichtum. Über die Runden kommen ist für sie schwer genug.

\section{Gini-Koeffizienten für die Reichen}

Der Gini-Koeffizient ist das bekannteste Maß zur Darstellung einer Verteilungssituation. Der Gini-Koeffizient ist sensibel gegenüber Veränderungen in der Mitte der Verteilung, nicht aber bei Veränderungen an den Rändern. Eine wirtschaftspolitische Orientierung am Gini-

II Aber auch in der Soziologie stellen die Arbeiten von Michael Hartmann eine Ausnahme dar (vgl. etwa Hartmann 2007).

I2 In den USA liefern das Forbes Magazine und Fortune solche Reihungen. In Großbritannien publiziert die Sunday Times seit 1989 jährlich eine Liste der Vermögenden. In Deutschland hat das Managermagazin eine Reihung der Reichen und in Österreich hat das Magazin Trend seit 200I jährlich eine solche Liste produziert. 
Koeffizienten muss vage bleiben, da es keine Vorstellung zu seiner optimalen Höhe gibt: Der Gini-Koeffizient der Geldvermögensverteilung in Österreich beträgt o,65 (vgl. Beer et al. 2006) ${ }^{13}$ In Österreich gab es 2004 circa 3.429.500 Haushalte. 2.556 Haushalte wurden in der OeNB-Vermögensumfrage 2004 befragt. Es existieren in Österreich momentan ca. 3.000 Privatstiftungen mit einem Geldvermögen von ca. 17,6 Milliarden $€$ (im Durchschnitt sind dies 5,9 Millionen $€$ ). Haushalte, die ihr Vermögen in einer Privatstiftung veranlagen, wurden in der OeNB-Umfrage nicht erfasst. Stellen wir nun folgendes Gedankenexperiment an: Um in der OeNB-Umfrage den Anteil von Stiftungen, hinter denen Haushalte stehen, angemessen abzubilden, müssten etwa zwei Stiftungen in die Stichprobe der OeNB aufgenommen werden. Da es zur Streuung des Vermögens bei den Privatstiftungen keine Informationen gibt, treffen wir die vorsichtige Hypothese, dass ein Haushalt in seiner Stiftung 5,9 Millionen $€$ hält und ein anderer 59 Millionen $€$. Bereits diese vorsichtige Annahme führt zu einer Erhöhung des Gini-Koeffizienten für die österreichische Geldvermögensverteilung von 0,65 auf 0,75 .

Doch auch der im Vergleich mit der Einkommensverteilung weit höhere Gini-Koeffizient repräsentiert nicht die Arm-Reich-Unterscheidung. Denn keine|r der reichsten Ioo Österreicher|innen wurde in die Umfrage einbezogen. Innerhalb dieser Gruppe beträgt der Gini-Koeffizient 0,67, d. h. sogar in einer so kleinen Gruppe ist die Ungleichheit zwischen Reichen und Superreichen enorm. Atkinson (2006) errechnet einen Wert von 0,46 für die Gruppe der Milliardär|inn|e|n.

Um die Spannweite der Vermögensverteilung in einer Maßzahl zu beschreiben, werden oft Perzentile verglichen. Bei P9o/Pro wird der untere Grenzwert des zehnten Dezils ins Verhältnis zum oberen Grenzwert des ersten Dezils gesetzt. Dieser Indikator ist aber unempfindlich gegenüber negativen Vermögenswerten im ersten Dezil und gegenüber Schwankungen der Werte des obersten Dezils. Bei P99/Pı ergibt sich zudem die Schwierigkeit, dass positives und negatives Nettovermögen nicht miteinander verglichen werden können. Beliebt ist daher, den Anteil des obersten Prozent (Promille) am Gesamtvermögen anzugeben. Da die nachfolgende Beurteilung, ob dieser Anteil niedrig oder hoch ist, nicht ohne subjektive Wertung zu einer gewünschten Verteilung getroffen werden kann, wird als Alternative entweder auf den Zeitverlauf oder auf einen Ländervergleich verwiesen.

Ein internationaler Vermögensreichtumsvergleich ist aber extrem schwierig (vgl. Sierminska et al. 2006a und 2006b, Ohlsson et al. 2006 und Davies et al. 2007b). Vermögensdaten kommen nicht nur aus einer Vielzahl von Quellen, zu unterschiedlichen Zeitpunkten, auf Basis unterschiedlicher Definitionen, sondern werden auch zu unterschiedlichen Zwecken von unterschiedlichen Institutionen erhoben. Jäntti/Sierminska (2007) zeigen auf Basis der aktuellen Ergebnisse der Luxembourg Wealth Studie (LWS) interessante Ergebnisse zur Vermögensungleichheit. ${ }^{14}$ Die Differenz zwischen Durchschnitt und Median des Netto-

I3 Davies et al. (2007b) schätzen für die Vermögensverteilung in Österreich einen Gini-Koeffizienten von 0,65 .

I4 Seit 7. Dezember 2007 liegen harmonisierte Vermögensdaten für zehn Länder (Deutschland, Finnland, Großbritannien, Italien, Kanada, Norwegen, Österreich, Schweden, USA und Zypern) vor, 
vermögens zeigt den höchsten Wert für Mexiko, gefolgt von den USA und Kanada. Die niedrigsten Werte weisen Schweden und Finnland auf. Dies weist auf die Bedeutung progressiver Vermögensbesteuerung hin.

Das harmonisierte LWS-Datenset (siehe Tabelle I) bereitet eine Reihe von Interpretationsschwierigkeiten und führt zu Zweifeln an der Datenqualität. Schweden weist im LWSDatenset einen vergleichsweise hohen Gini-Koeffizienten (o,89) auf. Dies ist in dem hohen Anteil an Haushalten mit einem negativen Nettovermögen begründet. Zieht man hingegen den Anteil des reichsten einen Prozents der Bevölkerung zur Beurteilung heran, dann liegen - wie erwartet - die USA mit deutlichem Abstand im Ländervergleich vorne.

\section{Tabelle I: Internationaler Vermögensverteilungsvergleich ${ }^{I}$}

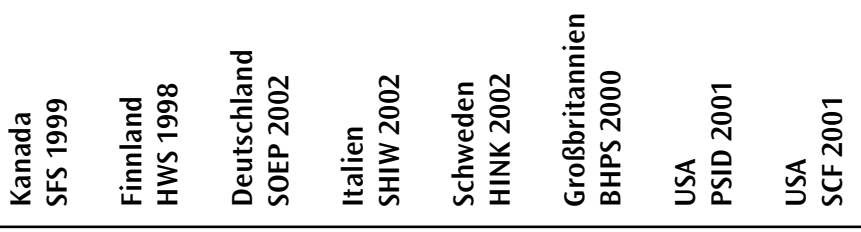

\begin{tabular}{|c|c|c|c|c|c|c|c|c|}
\hline Vermögensanteile & & & & & & & & \\
\hline Top $10 \%$ & 53 & 45 & 54 & 42 & 58 & 45 & 64 & 71 \\
\hline Top 5\% & 37 & 31 & 36 & 29 & 41 & 30 & 49 & 58 \\
\hline Top 1\% & 15 & 13 & 14 & 11 & 18 & 10 & 25 & 33 \\
\hline Ginikoeffizient & 0,75 & 0,68 & 0,78 & 0,61 & 0,89 & 0,66 & 0,81 & 0,84 \\
\hline \multicolumn{9}{|c|}{ Quantil/Median Ratio } \\
\hline 10. Perzentil & -17 & -6 & 0 & 0 & -84 & 0 & -11 & -15 \\
\hline 25. Perzentil & 0 & 1 & 0 & 8 & -1 & 2 & 0 & 0 \\
\hline 75. Perzentil & 350 & 218 & 886 & 209 & 447 & 238 & 378 & 368 \\
\hline 90. Perzentil & 708 & 390 & 1.818 & 359 & 972 & 482 & 925 & 980 \\
\hline
\end{tabular}

I Schwellenwert von 2.500€ für Vermögensaktiva und -passiva in Deutschland.

Quellen: LWS-Daten, Sierminska 2006a, SOEP-Daten

Davies et al. (2007a und 2007b) zeigen auf der Basis einer Reihe heroischer Annahmen, dass der Gini-Koeffizient weltweit im Jahr 2000 bei o,89 (auf Wechselkursbasis) und bei o,8o (auf Kaufkraftparitätenbasis) lag. Der Anteil der reichsten zehn Prozent der Bevölkerung lag

welche für Forscher|innen kostenfrei zugänglich sind http://www.lisproject.org/lws/lwsannouncement. htm. Das Projekt Luxembourg Wealth Study wurde im Jahr 2003 begonnen und ist nun eingebunden in die Luxembourg Income Study (LIS), die über 20 Jahre an Erfahrungen mit der Harmonisierung von Haushaltsbefragungsdaten zu Einkommen aufweist. 
bei 7I Prozent. Gefolgert werden kann eine extreme Ungleichverteilung, aber nichts über die Frage ihrer Rechtfertigung und damit über die wirtschafts- und gesellschaftspolitischen Implikationen (vgl. Schürz 2007).

\section{Haushaltsvermögensbefragungen: Mittelschichtforschung ohne Reiche}

Die Vielzahl von jüngst angelaufenen Haushaltsbefragungen zu Vermögen weckt die Hoffnung, dass nun bald Daten zu den Vermögensreichen zur Verfügung stehen werden. ${ }^{15}$ Diese Hoffnung scheint mir trügerisch. Da die Teilnahme an Vermögensbefragungen freiwillig erfolgt und Vermögensfragen, speziell von reichen Menschen, ungern beantwortet werden, finden sich selten Reiche in den repräsentativen Stichproben. Die Befragungsergebnisse weisen einen Mittelschichtsbias auf. Obdachlose und Anstaltsinsass|inn|en (Pflegeheime, Gefängnisse, Krankenhäuser) werden nicht erfasst und Reiche können kaum befragt werden.

Alle Haushaltsbefragungen zur Vermögenssituation sind mit dem Problem konfrontiert, dass Haushalte ihre Teilnahme vollständig verweigern oder auf bestimmte Fragen keine Antworten geben wollen. ${ }^{16}$ Problematisch ist hierbei, dass die Wahrscheinlichkeit der Verweigerung nicht als zufällig angesehen werden kann, sondern positiv von Einkommen und Vermögen abhängt. Meines Wissens wurde in keiner Haushaltsbefragung zum Vermögen jemals ein|e Milliardär|in befragt. Die wirtschaftspolitische Aussagekraft von Haushaltsbefragungen zu Reichtum ist daher vorab beschränkt.

Angesichts der starken Konzentration der Vermögen muss versucht werden, diesen Fehler zu korrigieren. Hierfür benötigt man eine spezielle Stichprobe, welche - etwa auf Basis von Steuerstatistiken (Vermögen- oder Einkommensteuer) oder auch unter Bedachtnahme auf geographische Charakteristika (wohlhabende Wohngegenden) oder Elektrizitätsverbrauch (Zypern) - die kleine Gruppe der Reichen extra zu erfassen sucht. ${ }^{17}$

Die Freiwilligkeit bei den Haushaltsbefragungen setzt der Reichtumsforschung enge Grenzen. Zur Erforschung des privaten Vermögensreichtums wäre staatlicher Zwang zur Informationsoffenlegung notwendig. Dass dies in vielen Ländern nicht einmal wirtschaftspolitisch gefordert wird, veranschaulicht die gesellschaftlichen Machtverhältnisse. Das Auslaufen von vermögensbezogenen Steuern - wie demnächst die Erbschaftssteuer in Österreich - bedeutet demnach stets neben dem öffentlichen Einnahmenverzicht auch einen

I5 Insbesondere Notenbanken engagieren sich aus Finanzmarktstabilitätsüberlegungen verstärkt bei der Erhebung von Mikrodaten zum Vermögen.

I6 In den meisten Haushaltsbefragungen werden Nichtbeantwortungen durch Imputationen korrigiert (vgl. Kennickell 2006).

I7 Es gibt mehrere Länder, die in ihren regelmäßigen Haushaltsbefragungen die Vermögenden gesondert zu erfassen suchen: Survey of Consumer Finances SCF (1983/1989/1992/1995/2001/2004) und Survey of Financial Security SFS (Kanada 1999; URL: http://www.statcan.ca/english/Dli/Data/Ftp/ sfs.htm), Survey of Consumer Finances CSCF (Zypern 1999/2002; URL: http://www.econ.ucy.ac.cy/ ${ }_{\%} 7$ Eechalias/survey.html), Household Wealth Survey (Finnland 1994/1998; URL: http:/www.stat. fi/tk/el/varallisuus.htmly und das Sozio-ökonomische Panel SOEP (Deutschland 1984-2003; URL: http://www.diw.de/deutsch/sop/). 
Verzicht auf Informationen zur ungleichen Verteilung. Mit Haushaltsbefragungen kann dieser Informationsverlust nicht wettgemacht werden.

In der Gesamtwirtschaftlichen Finanzierungsrechnung (GFR) werden regelmäßig Werte für das Geldvermögen der privaten Haushalte ermittelt. Problematisch ist hierbei u. a., dass im Zeitablauf teilweise unterschiedliche Abgrenzungen des Sektors der privaten Haushalte vorgenommen wurden und dass auch die Vermögenswerte von Stiftungen, Institutionen ohne Erwerbszwecke wie Nichtregierungsorganisationen und der Kirche enthalten sind.

Durch den Vergleich von GFR-Daten mit den Ergebnissen aus der Haushaltsbefragung können Messfehler zwar erkannt, aber nicht korrigiert werden. Ergibt sich in der Haushaltsbefragung etwa ein um 40 Prozent geringerer Wert an Aktien, so wäre es falsch, die angegebenen Werte mit 2,5 zu multiplizieren. Es könnte sein, dass die Befragten nur einen um 20 Prozent zu geringen Aktienwert bekannt gaben und der Rest sich aus einer fehlenden Beantwortung von Millionär|inn|en ergab. Der Aktienbesitz von Nichtmillionär|inn|en würde überhöht ausgewiesen werden.

Die statistischen Beschränkungen der Haushaltsbefragungen zu privatem Vermögen verlangen, den Geltungsbereich der Vermögensbefragungen für wirtschaftspolitische Interpretationen vorab einzuschränken (z. B. die Ergebnisse nur als eine Informationsquelle für Vermögen bis zehn Millionen $€$ zu verstehen).

\section{Arme arm machende Reiche}

Die Schwellenwerte, ab wann von Reichtum zu sprechen ist, bleiben notwendigerweise arbiträr und normativ zudem gehaltlos. Die Versuche, Reichtum analog zu Armut zu messen, müssen analytisch ebenso scheitern wie jene der offiziellen Armutsmessung. Denn die Armutsgefährdung quantifiziert nicht nur in willkürlicher Form den Abstand vom Median, sondern im Median kulminieren auch keine gesellschaftlichen Entwicklungen. ${ }^{\text {I8 }}$ Insbesondere reflektiert der Median nicht die Einkommensentwicklungen am rechten Ende der Verteilung bei den Reichsten.

Für den Marxismus waren Armut und Reichtum Pol und Gegenpol der kapitalistischen Produktion. ${ }^{19}$ In einem Bourdieuschen Verständnis ginge es um soziale Distanz von Akteuren in einem Feld (vgl. Schürz 2008a). Gesellschaftstheoretisch gestützte Versuche einer gleichzeitigen Konzeptualisierung von Arm/Reich sind heute jedoch selten geworden.

Gerade in aktuellen gesellschaftspolitischen Debatten wird das Thema der sozialen Ungleichheit in unterschiedliche Fragen arbeitsteilig zerlegt. Man konzentriert sich etwa auf Kinderarmut oder Exzesse beim Managementeinkommen und vernachlässigt bzw. negiert das bekannte Brechtsche Diktum: „Wär' ich nicht arm, wärst du nicht reich.»

I8 Als armutsgefährdet oder von Armutsrisiko betroffen werden jene Personen bezeichnet, deren äquivalisiertes Haushaltseinkommen unter einer Armutsgefährdungsschwelle von 60 Prozent des Median liegt.

I9 Für eine konzise Übersicht zur marxistischen Perspektive auf Armut/Reichtum siehe TjadenSteinhauer (1996) im Historisch-Kritischen Wörterbuch des Marxismus. 
Auch ergeben sich durch Perspektiveinengung leicht erkennbare inkonsistente Argumentationen. Zwei Beispiele: Bei einer normativen Beurteilung von Armut wird oft die individuelle Verantwortung befragt, also die Frage, ob die Armen an ihrem Schicksal selbst schuld seien. Im weit verbreiteten Glücksegalitarismus soll etwa nur für unverschuldetes Leid eine Kompensation geleistet werden. Was Menschen hingegen selbst zu verantworten haben, soll auch gesellschaftlich nicht entschädigt werden. Bei den Reichen läuft die aktuelle neoliberale Argumentation anders. Auch bei unverschuldetem Reichtum, der ohne eigenes Zutun erworben wird (Erbschaften, Schenkungen, Börsengewinne, Immobilienwertsteigerungen), soll der Staat keinesfalls massiv besteuern. Ein anderes Beispiel inkohärenter neoliberaler Argumentation ist das geläufige Diktum, die Reichen müssten reicher werden (sonst investierten sie nicht) und die Armen müssten ärmer werden (sonst arbeiteten sie nicht).

Der Willkür und normativen Bedeutungslosigkeit von statistischen Schwellenwerten für Armut und Reichtum könnte durch eine Rückkoppelung des Begriffpaares Arm / Reich an das Gemeinwesen entgangen werden. Im Gemeinwesen gelten bestimmte politische Gleichheitsideen und Vorstellungen von gleichen sozialen Teilhabemöglichkeiten. Massive Armut, aber auch exzessiver Reichtum gefährden eine Gesellschaft in ihrem Zusammenhalt (vgl. Pogge 2007, Andreß / Kronauer 2007). Während Armut geringere soziale Teilhabe und geringere politische Mitgestaltung impliziert, bedeutet Reichtum ein Übermaß an politischen Einflussmöglichkeiten. Reiche können sich freiwillig sozial ausgrenzen via Privatschulen, privater Gesundheitsvorsorge und privat gesicherten Wohngegenden. Und eine Vermögenskonzentration impliziert Konzentration von Macht und damit einhergehend die Möglichkeit, demokratische Institutionen inhaltlich auszuhöhlen.

Das Gemeinsame beider sozialer Randpositionen der Verteilung, Arm und Reich, ist, dass sie aus einer normativen politischen Gleichheitsperspektive negativ zu beurteilen sind. Für die Reichtumsforschung bedeutet dies, dass Indikatoren kohärent für die gesamte soziale Verteilung, von Arm bis Reich, entwickelt werden müssen. Den nichtmonetären Komponenten (soziales Kapital, kulturelles Kapital, Habitus, Vererbung von Ressourcen usw.) ist in ihrer Verflechtung mit monetären Ressourcen (Einkommen, Vermögen) ein besonderes Augenmerk zu schenken. Die Missbrauchsmöglichkeiten von Reichtum könnten über Lobbyingaktivitäten (Parteispenden), geringere Distanz zu politischen Entscheidungsträger|inne|n (Sozialkontakte), Biographien von Ex-Politiker|inne|n empirisch untersucht werden (vgl. Jacobs/Skocpol 2005). Datenbeschränkungen zu den Eliten setzen einem solchen Unterfangen zwar enge Grenzen, doch gerade dieses Faktum unterstreicht die gesellschaftspolitische Bedeutung einer theoriegeleiteten empirischen Reichtumsforschung.

Die Frage nach der Entstehung von Reichtum (legal, erarbeitet, geerbt, usw.) kann historisch rekonstruiert werden (vgl. etwa Phillips 2002, Keister 2005 und Rubinstein 2006). Ihre Beantwortung erlaubt ein normatives Urteil über die Reichen. Bereits Aristoteles hatte aber auf eine weitere wichtige Unterscheidung aufmerksam gemacht. Von Reichtum kann in Bezug auf letzte Zwecke oder in Bezug auf Mittel die Rede sein: 
„Das auf Gelderwerb gerichtete Leben hat etwas Unnatürliches und Gezwungenes an sich, und der Reichtum ist das gesuchte Gut offenbar nicht. Denn er ist nur für die Verwendung da und nur Mittel zum Zweck." (Aristoteles I995: 6)

Während der Frage, wer die Reichen sind, viel Aufmerksamkeit geschenkt wird, ist jene nach der Verwendung von Reichtum wenig erforscht. Ob Reichtum im Rahmen der geltenden Gesetze, unter Beachtung demokratischer Spielregeln verwendet wird, wissen wir nicht. Die Frage, ob Steuerhinterziehungen Reicher häufiger passieren als Sozialhilfemissbrauch durch Arme, wird nur selten überhaupt analysiert (vgl. Krysmanski 200I). Dies mag damit zusammenhängen, dass

»die wirklichen Eliten sich dadurch aus[zeichnen], dass sie in der Lage sind, qua Position maßgeblichen Einfluss auf gesellschaftliche Entwicklungen zu nehmen.« (Hartmann 2007: I8)

Es gibt nur Reichtum, der in Zusammenhang mit Armut steht. Sinken die sozialen Bindungskräfte in einer Gesellschaft, die wenigstens ideologisch dereinst suggerierte, dass es nach allgemein anerkannten Prinzipien der Verteilungsgerechtigkeit zugehe, dann führt Reichtumskonzentration zu einer Gesellschaft, die zwischen Oben und Unten antagonistisch zerrissen ist (vgl. Negt 2005). Von Armut unabhängiger Reichtum ist ein Artefakt. Auch bei Bildung und Gesundheit erfolgt Privilegierung stets auf Kosten von anderen. Eine Vorreihung bei Operationsterminen verschlechtert die Chancen von weniger Privilegierten und ein Besuch bestimmter elitärer Bildungsinstitutionen schmälert vorab die Aufstiegschancen von Arbeiterkindern. Gesellschaftlicher Reichtum, Schulen, Krankenhäuser, Flüsse, Wälder und Luft können privaten Reichtum ergänzen, werden aber auch von diesem bedroht. Wenn weiterhin nur ein geringer Prozentsatz an Arbeiterkindern auf die Universität geht, dann müsste auch die öffentliche Universität zu einem Teil dem privaten Reichtum zugerechnet werden. Denn Teile des öffentlichen Reichtums sind Subventionen an Wohlhabende.

Reichtumsforschung dient der Befragung sozialer Unterschiede. Ideologisch wird Reichtumsforschung für die Legitimierung sozialer Distanz instrumentalisiert. Sie rechtfertigt sie, indem sie positive Funktionen privaten Reichtums für die Gesellschaft hervorzuheben sucht. Und sie leistet erst dann einen gesellschaftskritischen Beitrag, wenn sie die ungerechtfertigten Privilegien der Reichen demaskiert.

\section{Schlussfolgerungen}

Meine erste Annäherung an eine Definition von Reichen wäre gewesen, dass es sich hierbei um jene Menschen handle, die sich problemlos der Frage nach ihren Vermögensverhältnissen entziehen können. Doch gibt diese Definition keinen Aufschluss darüber, ob die Verschwiegenheit der Reichen problematisch oder nur eine Idiosynkrasie ist.

In der Argumentation des Papiers wurden konzeptuelle Schwierigkeiten der Reichtumsforschung aufgezeigt. Diese reichen von statistischen Unzulänglichkeiten über normative Unklarheiten bis zu den Eigeninteressen der Wohlhabenden, die eine angemessene 
wissenschaftliche Forschung erschweren. Werturteilsfrei kann nicht bestimmt werden, was unter Reichtum zu verstehen ist, denn objektive Reichtumsmaße gibt es nicht. Besondere Probleme stellen sich bei der Erfassung der nicht-monetären Komponenten von Reichtum. Argumentiert wurde für eine relationale Sichtweise, die sich dem Arm-Reich-Thema gemeinsam zu nähern sucht. Die Arm-Reich-Distinktion wurde auf das politische Gemeinwesen bezogen. Denn noch besteht ein breiter gesellschaftlicher Konsens hinsichtlich der Bedeutung von politischer Gleichheit. Wenigstens ideologisch wird Gesellschaft noch als Gesellschaft von Gleichen verstanden, die einander alle etwas schulden. Von den Armen wird sie aber längst nicht mehr als solche erlebt, und dies zeigt an, dass die Reichtumskonzentration die Gesellschaft zerfrisst. Die gesellschaftlichen Verhältnisse erleben eine schleichende Refeudalisierung.

\section{Literatur}

Andreß, Hans-Jürgen / Kronauer, Martin (2007): Arm - Reich, in: Lessenich, Stephan / Nullmeier, Frank (Hg.), Deutschland eine gespaltene Gesellschaft, Frankfurt am Main, S. 28-53

Aristoteles (1995): Philosophische Schriften, Band 3, Hamburg

Atkinson, Anthony B./Gordon, James/Harrison, Alan (1989): Trends in the Shares of Top

Wealthholders in Britain: 1923-1989, in: Oxford Bulletin of Economics and Statistics, Jg. 51, Nr. 3, S. 315-332

Atkinson, Anthony B. (2006): Concentration Among the Rich, Research Paper presented at the UNU-WIDER Project Meeting, Nr. 2006/I5I

Beer, Christian / Mooslechner, Peter / Schürz, Martin / Wagner, Karin (2006): Das Geldvermögen privater Haushalte in Österreich: Eine Analyse auf Basis von Mikrodaten, in: Geldpolitik und Wirtschaft, Nr. 2, S. IOI-II9

Davies, James/Shorrocks, Anthony (2000): The Distribution of Wealth, in: Atkinson, Anthony B. / Bourguignon, Francois (Hg.), Handbook of Income Distribution, Volume I, Amsterdam: Elsevier, S. 605-675

Davies, James/Sandstrom, Susanna/Shorrocks, Anthony/Wolff, Edward (2007a): The World Distribution of Household Wealth, URL: http://repositories.cdlib.org/cgi/viewcontent. cgi? article $=$ I068 $\&$ context $=$ cgirs

Davies, James/Sandstrom, Susanna/Shorrocks, Anthony/ Wolff, Edward (2007b): Estimating the Level and Distribution of Global Houshold Wealth, UNU-WIDER Research Paper, Nr. $2007 / 77$

Deutsche Bundesregierung (2005): Lebenslagen in Deutschland, 2. Armuts- und Reichtumsbericht, Berlin

Grabka, Markus / Frick, Joachim (2007): Vermögen in Deutschland wesentlich ungleicher verteilt als Einkommen, in: DIW-Wochenbericht, Jg. 2007, Nr. 45, S. 665-672

Ehrenreich, Barbara (2007): The Rich are Making the Poor Poorer, in: The Nation, I3. Juni 2007, URL: http://www.alternet.org/workplace/53962/

Ermisch, John/Francesconi, Marco/Siedler, Thomas (2006): Intergenerational Mobility and Marital Sorting, in: The Economic Journal, Jg. II6, Nr. 513, S. 659-679 
Faik, Jürgen (200I): Empirische Befunde zur Entwicklung der Vermögensverteilung privater Haushalte in Deutschland, in: Stadlinger, Joachim (Hg.), Reichtum heute. Diskussion eines kontroversen Sachverhalts, Münster, S. 68-80

Hartmann, Michael (2007): Eliten und Macht in Europa. Ein internationaler Vergleich, Frankfurt am Main

Huster, Ernst-Ulrich (1997) (Hg.): Reichtum in Deutschland. Die Gewinner der sozialen Polarisierung, Frankfurt am Main

Huster, Ernst-Ulrich (200I): Reichtum in Deutschland. Die Gewinner der sozialen Polarisierung, in: Stadlinger, Joachim (Hg.), Reichtum heute. Diskussion eines kontroversen Sachverhalts, Münster, S.9-28

ISG (2007): Weiterentwicklung der Reichtumsberichterstattung der Bundesregierung. ExpertenWorkshop am 29. November 2006 in Berlin, URL: http://www.bmas.de/coremedia/generator /908/property=pdf/lebenslagen__in__deutschland__reichtumsberichterstattung__ expertenworkshop.pdf

Jacobs, Lawrence/ Skopcol, Theda (2005): Inequality and American Democracy, New York: The Russell Sage Foundation

Keister, Lisa (2005): Getting Rich. America's New Rich and How They Got That Way, Cambridge: Cambridge University Press

Kennickell, Arthur (2006): Currents and Undercurrents: Changes in the Distribution of Wealth, 1989-2004, URL: http://www.ombwatch.org/budget/pdf/FRBWealthreport. pdf

Klevmarken, Anders (2003): On Household Wealth Trends in Sweden over the I990s, The Levy Economics Institute Working Paper, Nr. 395

Kopczuk, Wojciech/Saez, Emmanuel (2004): Top Wealth Shares in the United States, 1916-2000: Evidence from Estate Tax Returns, in: National Tax Journal, Jg. 57, Nr. 2, S. 445-487

Krysmanski, Hans Jürgen (200I): Der stille Klassenkampf von oben. Strukturen und Akteure des Reichtums, in: UTOPIE kreativ, Nr. 205, S. 999-IOII

Mäder, Ueli/Streuli, Elisa (2002): Reichtum in der Schweiz, Portraits - Fakten - Hintergründe, Zürich

Merrill Lynch/Capgemini (2007): World Wealth Report, URL: http:/www.ml.com/media/ 79882.pdf

Negt, Oskar (2005): An Heinrich von Pierer. Von der Wirtschaft gegen den Menschen, in: Amery, Jean (Hg.); Briefe an den Reichtum, München, S. 6o-85

Ohlsson, Henry/Roine, Jesper/ Waldenström, Daniel (2006): Long-run Changes in the Concentration of Wealth, UNU-WIDER Research Paper, Nr. 2006/IO3

Phillips, Kevin (2002): Wealth and Democracy. A Political History of the American Rich, New York: Broadway Books

Piketty, Thomas / Postel-Vinay, Gilles / Rosenthal, Jean-Laurent (2006): Wealth Concentration in a Developing Economy: Paris and France, 1807-1994, in: American Economic Review, Jg. 96, Nr. I, S. 236-256

Pogge, Thomas (2007): Why Inequality Matters, in: Held, David/Kaya, Ayse (Hg.), Global Inequality: Patterns and Explanations, Cambridge: Polity Press, S. 132-I47 
Rubinstein, William (2006): Men of Property. The Very Wealthy in Britain Since the Industrial Revolution, London: The Social Affairs Unit

Schürz, Martin (2007): Ungleich in der Ungleichheit. Gedankensplitter zur ungerechtfertigten Ungleichheit, in: Rosecker, Michael/Müller, Bernhard (Hg.), Gleichheit, Wiener Neustadt, S. 85-IO4

Schürz, Martin (2008a): Pierre Bourdieus Ungleichheitssoziologie und Amartya Sens Fähigkeitenansatz: Unterschiedliche Perspektiven auf gesellschaftliches Leid, in: Kurswechsel, Nr. I, S. 46-55

Schürz, Martin (2008b): Anmerkungen zur Messung des Vermögen privater Haushalte in: Zeitschrift für angewandte Sozialforschung, Jg. 25, Nr. I/2, S. I37-I48

Sierminska, Eva/ Brandolini, Andrea/Smeeding, Tim (2006): The Luxembourg Wealth Studya Cross-Country Comparable Database for Household Wealth Research, in: Journal of Economic Inequality, Jg. 4, Nr.3, S. 375-383

Stadlinger, Joachim (Hg.) (200I): Reichtum heute. Diskussion eines kontroversen Sachverhalts, Münster

Stein, Holger (2004): Anatomie der Vermögensverteilung, Ergebnisse der Einkommens- und Verbraucherstichproben $1983-1998$, Berlin

Tjaden-Steinhauer, Margarete (1996): Armut / Reichtum, in: Haug, Wolfgang F. (Hg.), HistorischKritisches Wörterbuch des Marxismus Band I, Berlin, Sp. 608-6i3

Volkert, Jürgen (2005) (Hg.): Armut und Reichtum an Verwirklichungschancen, Wiesbaden 